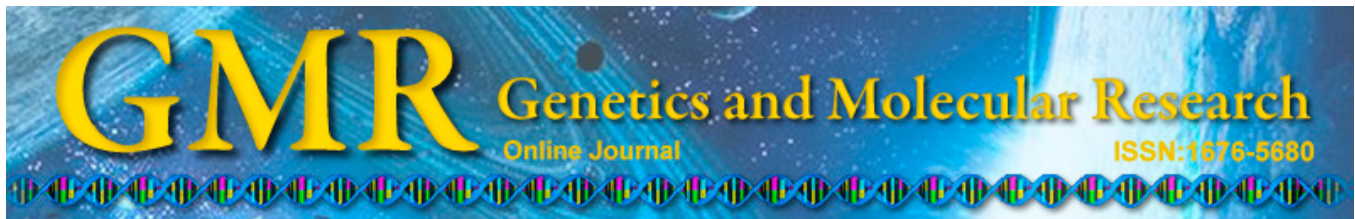

\title{
Regulation of the expression of zinc finger protein genes by microRNAs enriched within acute lymphoblastic leukemia-derived microvesicles
}

\author{
L. Lu ${ }^{1 *}$, X.M. Chen ${ }^{1 *}$, H.M. Tao ${ }^{1}$, W. Xiong ${ }^{1}$, S.H. Jie ${ }^{2}$ and H.Y. Li ${ }^{1}$ \\ ${ }^{1}$ Center for Stem Cell Research and Application, Institute of Hematology, \\ Union Hospital, Tongji Medical College, \\ Huazhong University of Science and Technology, Wuhan, China \\ ${ }^{2}$ Department of Infectious Diseases, Union Hospital, Tongji Medical College, \\ Huazhong University of Science and Technology, Wuhan, China \\ *These authors contributed equally to this study. \\ Corresponding author: H.Y. Li \\ E-mail: huiyuli26@aliyun.com
}

Genet. Mol. Res. 14 (4): 11884-11895 (2015)

Received February 10, 2015

Accepted June 29, 2015

Published October 5, 2015

DOI http://dx.doi.org/10.4238/2015.October.5.2

\begin{abstract}
Microvesicles (MVs) are submicrometric membrane fragments that can "engulf" cytoplasmic contents such as microRNAs (miRNAs) from their cellular origin. The study of miRNAs carried within MVs might provide insights into the roles that miRNAs play in the underlying pathophysiologic processes of acute lymphoblastic leukemia (ALL). We identified numerous dysregulated MV miRNAs in patients with B- and T-cell ALL by using Agilent microarray analysis. Selected miRNAs obtained by microarray profiling were validated using quantitative reverse transcription-polymerase chain reaction. Using bioinformatic tools, we found that 118 and 116 miRNAs from Band T-ALL MVs, respectively, regulated the expression of zinc finger protein (ZFP) genes. For example, zinc finger protein 238 (ZNF238),
\end{abstract}


known as a tumor suppressor, was regulated by miR-20b over-expression. Conversely, ZNF267, a cancer-promoting factor, was mediated by downregulated miR-23a and miR-23b. Considering that miRNAs are generally believed to repress gene expression, antineoplastic ZNF238 was likely inhibited while the level of oncogenic ZNF267 was likely increased by miRNA dysregulation, leading to modification of the ALL microenvironment. In addition, gene ontology and signaling pathway analysis demonstrated that a subset of the ZFP genes targeted by altered MV miRNAs are involved in cellular biological processes including proliferation, differentiation, apoptosis, and cell cycle regulation. These findings indicated that cancer-associated MV miRNAs and their target ZFP genes might be novel pathogenic factors in ALL. However, the specific roles exerted by MV miRNAs and their target ZFP genes on the pathological mechanisms of ALL remain to be further understood.

Key words: Microvesicles; MicroRNAs; Zinc finger protein genes; Acute lymphoblastic leukemia

\section{INTRODUCTION}

Leukemia, also known as "blood cancer" is characterized by malignant cloning of hematopoietic stem cells and developmental arrest of immature leukocytes in diverse phases. Presently, it is estimated that over 2.6 million patients die of leukemia annually worldwide (Ferlay et al., 2013). Of the major types of leukemia, acute lymphoblastic leukemia (ALL) mostly strikes children, accounting for nearly $80 \%$ of childhood acute leukemia. Possessing aberrant abilities including enhanced self-renewal and incontrollable proliferation, primitive and immature lymphoid cells accumulate extensively in bone marrow, lymphoid tissues, and other organs, resulting in normal hematopoietic function being inhibited and the failure of infiltrated organs. Mounting evidence has established that the pathogenic events of ALL involve genetic and epigenetic abnormalities induced by factors such as chromosomal translocation and ionizing radiation (Pui et al., 2008). Given that leukemogenesis is an intricate and complex process, the specific pathological mechanisms of ALL remain to be further investigated.

Microvesicles (MVs) are submicrometric vesicular structures of cytoplasmic membrane origin that are shed by various types of cells such as leukemic cells (Li et al., 2014; Zhang and Grizzle, 2014). These nano-sized sacs can "hijack" membrane components and engulf cytoplasmic contents from their cellular origin. MVs are enriched with a variety of bioactive molecules from their parental cells including lipids, proteins, and nucleic acids [DNA, mRNA, and microRNA (miRNA)] (Pisetsky et al., 2011; Raposo and Stoorvogel, 2013). It is well documented that MVs have significant roles in intercellular communication through transference of cargos packaged within MVs locally or systemically (Cocucci et al., 2009).

Over the past years, increasing evidence has indicated that altered expression profiles of miRNAs are present in several diseases, in particular in malignant neoplasms (Ikeda and Tagawa, 2014; Srivastava et al., 2014), suggesting that miRNAs are involved in the pathogenesis of malignancies. As pivotal regulators of gene expression at the post-transcriptional level, miRNAs are capable of binding to the 3'-untranslated regions (3'-UTRs) of target mRNAs, 
inducing the repression of mature mRNA molecular levels (Guo et al., 2010). Currently, it is believed that miRNAs could act as oncogenes or tumor suppressor genes; miRNAs have been shown to be associated with tumor progression, such as in tumor cell proliferation, evasion from apoptosis, invasion, and metastasis (Negrini et al., 2009). Given that MVs are able to work as cell-to-cell communication tools for transferring bioactive materials like miRNAs between cells (Valadi et al., 2007), MVs released by leukemia cells constitute an important part of the leukemia microenvironment. However, little is known about the miRNAs contained within MVs from leukemia cells. In this study, we investigated the miRNA expression signatures of ALL-derived MVs and explored the putative roles of MV miRNAs in the pathophysiological processes of leukemia.

\section{MATERIAL AND METHODS}

\section{Sample collection}

We analyzed the peripheral blood specimens from newly diagnosed patients with T-cell acute lymphoblastic leukemia (T-ALL) and B-cell acute lymphoblastic leukemia (B-ALL) and from healthy volunteers. Samples of patients diagnosed with T-ALL or B-ALL were obtained from the Wuhan Union Hospital, Tongji Medical College, Huazhong University of Science and Technology from June to December 2013. Diagnosis of ALL was based on morphologic, immunologic, cytogenetic, and molecular biologic (MICM) criteria as previously described (Vitale et al., 2006). The characteristics of patients and volunteers are listed in Table 1. In addition, the criteria for healthy volunteers consisted of no recent illnesses or treatments for a chronic medical condition. No medical history was obtained from the donors. Our study was approved by the Ethics Committee of Wuhan Union Hospital. All subjects provided written informed consent before entry into the study. Blood samples were collected at 8:00 am.

\begin{tabular}{|c|c|c|c|c|c|c|c|c|}
\hline \multirow[t]{2}{*}{ Group } & \multirow[t]{2}{*}{ No. } & \multicolumn{2}{|c|}{ Gender } & \multicolumn{2}{|c|}{ Age } & \multirow[t]{2}{*}{ WBC $\left(\times 10^{9} / \mathrm{L}\right)$} & \multirow[t]{2}{*}{$\operatorname{PLT}\left(\mathrm{x} 10^{9} / \mathrm{L}\right)$} & \multirow[t]{2}{*}{$\mathrm{Hb}(\mathrm{g} / \mathrm{L})$} \\
\hline & & F & M & Median & Range & & & \\
\hline B-ALL & 46 & 29 & 17 & $41.52 \pm 3.45$ & $10-66$ & $26.81 \pm 5.64$ & $103.21 \pm 7.81$ & $120.42 \pm 13.51$ \\
\hline T-ALL & 43 & 22 & 21 & $35.52 \pm 3.74$ & $8-55$ & $28.77 \pm 6.65$ & $110.35 \pm 5.36$ & $121.57 \pm 24.66$ \\
\hline Controls & 41 & 25 & 16 & $45.32 \pm 1.68$ & $13-52$ & $7.53 \pm 1.31$ & $189.35 \pm 7.32$ & $135.45 \pm 14.22$ \\
\hline
\end{tabular}

$\mathrm{B}-\mathrm{ALL}=\mathrm{B}$-cell acute lymphoblastic leukemia; T-ALL $=$ T-cell acute lymphoblastic leukemia; $\mathrm{WBC}=$ white blood cells; $\mathrm{PLT}=$ platelets; $\mathrm{Hb}=$ hemoglobin.

\section{Isolation of MVs}

Samples from patients with ALL and from donors were collected in potassium diamine ethylene acetic tetra acid (K $\mathrm{K}_{2}$ EDTA)- containing tubes. Peripheral blood was centrifuged at $400 \mathrm{~g}$, and blood plasma was isolated from whole blood stored on ice and was further gathered for MV preparation. Cell debris in plasma was removed by centrifugation at $2500 \mathrm{~g}$ for $30 \mathrm{~min}$. The obtained supernatants were ultracentrifuged at $16,000 \mathrm{~g}$ for $120 \mathrm{~min}$ to purify MVs. Subsequently, MV pellets were washed with cold phosphate-buffered saline containing $5 \mathrm{mM}$ 4-(2-hydroxyethyl)-1-piperazineethanesulfonic acid (HEPES) and then treated with 
RNase to degrade any remaining extracellular RNAs to guarantee that all RNAs detected were of MV origin. MV pellets were resuspended in lysate and stored at $-80^{\circ} \mathrm{C}$ until used. All centrifugations were performed at $4^{\circ} \mathrm{C}$.

\section{RNA extraction and purification}

Total RNAs were isolated from B-ALL- and T-ALL-derived MVs and from control MVs using the mirVana microRNA isolation kit according to manufacturer instructions (Ambion, Austin, TX, USA). The RNA qualities, yields, and sizes of RNA fractions were analyzed using an Agilent 2100 Bioanalyzer (Agilent Technologies, Santa Clara, CA, USA). For RNA isolated from MVs, only samples with an RNA integrity number $\geq 6.0$ and $28 \mathrm{~S} / 18 \mathrm{~S} \geq 0.7$ were used for further profiling (Table 2).

Table 2. Quality control of RNA samples extracted from MVs of the three groups.
\begin{tabular}{lccccr}
\hline Sample name & Concentration $(\mu \mathrm{g} / \mu \mathrm{L})$ & Volume $(\mu \mathrm{L})$ & $\mathrm{A}_{260} / \mathrm{A}_{280}$ & \multicolumn{2}{c}{ Agilent 2100 Results } \\
\cline { 3 - 5 } & & & $2.19 \pm 0.04$ & $\mathrm{RIN}$ & $28 \mathrm{~S} / 18 \mathrm{~S}$ \\
\hline B-ALL-MVs & $0.133 \pm 0.005$ & 100 & $2.16 \pm 0.06$ & $1.8 \pm 0.2$ \\
T-ALL-MVs & $0.109 \pm 0.012$ & 100 & $1.98 \pm 0.3$ & $1.4 \pm 0.4$ \\
Control-MVs & $0.084 \pm 0.009$ & 100 & $1.98 \pm 0.03$ & $6.7 \pm 0.6$ & $1.4 \pm 0.2$ \\
\hline
\end{tabular}

$\mathrm{MVs}=$ microvesicles; B-ALL = B-cell acute lymphoblastic leukemia; T-ALL = T-cell acute lymphoblastic leukemia; $2100=$ Agilent 2100 Bioanalyzer; RIN $=$ RNA integrity number.

\section{miRNA microarray analysis}

miRNAs in total RNA were labeled using an miRNA Complete Labeling and $\mathrm{Hyb}$ Kit (Cat. \#5190-0456, Agilent Technologies); each slide was hybridized with 100 ng Cy3labeled RNA in a hybridization oven (Cat. \#G2545A, Agilent Technologies) at $55^{\circ} \mathrm{C}$, with 20 $\mathrm{rpm}$ rotation for $20 \mathrm{~h}$ according to manufacturer instructions. After hybridization, slides were washed in staining dishes (Cat. \#121, Thermo Shandon, Waltham, MA, USA) using a Gene Expression Wash Buffer Kit (Cat. \#5188-5327, Agilent Technologies). Slides were scanned by an Agilent Microarray Scanner (Cat. \#G2565BA, Agilent Technologies) with the Feature Extraction software 10.7 (Agilent Technologies) with default settings. Raw data were exported directly into the GeneSpring GX11.0 software (Agilent Technologies) for quartile normalization and further analyses.

\section{Statistical analysis}

All data are reported as means and standard derivations. After the raw data of every miRNA in each group were background corrected, normalized, and averaged, we used the Significance Analysis of Microarray software (SAM, Stanford University, Stanford, CA, USA) for statistical comparisons. Differences were deemed significant at a $\mathrm{P}$ value $<0.05$. Statistical analysis was carried out using the R-software (The R Project for Statistical Computing, http:// www.r-project.org/). We compared the profiles of miRNAs between groups quantitatively by fold-change value. After the original data from all chips were standardized, fold-change was calculated as the ratio between signals of the miRNA in two different groups. miRNAs with $\mathrm{P}$ values $<0.05, \mathrm{FC}<0.5$ or $\mathrm{P}$ values $<0.05, \mathrm{FC}>2$ were subjected to further analysis. 


\section{Validation of microarray data}

For testing of candidate miRNAs acquired from the microarray analysis, quantitative reverse transcription-polymerase chain reaction (qRT-PCR) was performed. cDNA was synthesized from total RNA using the PrimeScript RT Reagent Kit (TaKaRa Bio, Otsu, Shiga, Japan) according to the manufacturer protocol. The reaction was incubated for $15 \mathrm{~min}$ at $37^{\circ} \mathrm{C}$, $5 \mathrm{~s}$ at $85^{\circ} \mathrm{C}$ and then kept at $4^{\circ} \mathrm{C}$. mRNA was quantified using an SYBR Green PCR master mix and an ABI 7500 PCR system (Applied Biosystems, Inc., Foster City, CA, USA). The reaction was incubated at $95^{\circ} \mathrm{C}$ for $30 \mathrm{~s}$, followed by 40 cycles of $5 \mathrm{~s}$ at $95^{\circ} \mathrm{C}$ and $30 \mathrm{~s}$ at $60^{\circ} \mathrm{C}$. All assays were repeated three times. The miRNAs for qRT-PCR consisted of miR-1, miR1301, miR-335, miR-889, miR-1290, miR-1268, miR-765, and miR-630. The small nuclear RNA U6 was used as an internal control. Primers for qRT-PCR are shown in Table 3. Data for qRT-PCR were analyzed using the comparative CT method, which was normalized against the expression of U6.

\begin{tabular}{ll} 
Table 3. Details for qRT-PCR primer sequences. \\
\hline miRNA & Primer sequence \\
\hline U6 Forward & CGCTTCGGCAGCACATATAC \\
U6 Reverse & TTCACGAATTTGCGTGTCAT \\
miR-1 Forward & CTGTCACTCGAGCTGCTGGAATG \\
miR-1 Reverse & ACCGTGTCGTGGAGTCGGCAATT \\
miR-1301 Forward & TTGCAGCTGCCTGGGAGTGACTTC \\
miR-1301 Reverse & AGTCACCTCCCAGGCAGCTGCAATT \\
miR-335 Forward & TCAAGAGCAATAACGAAAAATGT \\
miR-335 Reverse & GCTGTCAACGATACGCTACGT \\
miR-889 Forward & GCTTAAAGAATGGCTGTCCGTAG \\
miR-889 Reverse & GATACTAAAACAATGGTTGTCCGA \\
miR-1290 Forward & TACCTCTGCCTGCTGAAAGC \\
miR-1290 Reverse & CTCGAAGTGGCCAGTGTCTT \\
miR-1268 Forward & CTCAGCCTCCAAAGTAGCTG \\
miR-1268 Reverse & ATCGGCTCGCACCACCATTT \\
miR-765 Forward & GCCCTTTTCAAGCCCTACGA \\
miR-765 Reverse & GGCTCACGAGAACAGATCCT \\
miR-630 Forward & ACTTAACATCATGCTACCTCTTTGT \\
miR-630 Reverse & AGAACTACCTTCCCTGGTACAGA \\
\hline
\end{tabular}

\section{Bioinformatic analysis}

We performed the prediction of miRNA targets by using computational algorithms according to the base-pairing rules between miRNAs and target sites within the 3'-UTRs of target mRNAs. In our study, we primarily utilized TargetScan v6.2 (http://www.targetscan.org/) to predict potential target genes of the aberrantly expressed miRNAs. Gene ontology (GO) annotation and signaling pathway analysis were performed to investigate the candidate target genes.

\section{RESULTS}

\section{Presence of dysregulated miRNAs in MVs derived from patients with ALL}

We identified the expression signatures of MV miRNAs in patients with B-ALL and T-ALL and in the normal donors. With microarray analysis, numerous aberrantly expressed 
miRNAs were found in MVs derived from patients with B-ALL and T-ALL compared with those from the controls. Of the human miRNAs detected, 333 dysregulated miRNAs $(\mathrm{P}<$ $0.05)$ were present in B-ALL-derived MVs and $371(\mathrm{P}<0.05)$ were identified in T-ALL-MVs. Specifically, there were 136 upregulated and 197 downregulated miRNAs in B-ALL MVs. Comparably, 139 miRNAs carried inside T-ALL-MVs were found to be highly expressed and 232 were poorly expressed, as defined by expression levels of over 2 -fold or under 0.5 -fold difference from controls. We further observed that there were 107 co-upregulated and $180 \mathrm{co}-$ downregulated MV miRNAs among B-ALL and T-ALL samples. Figure 1 shows the altered miRNAs with diverse expression levels in the two types of ALL-MVs.
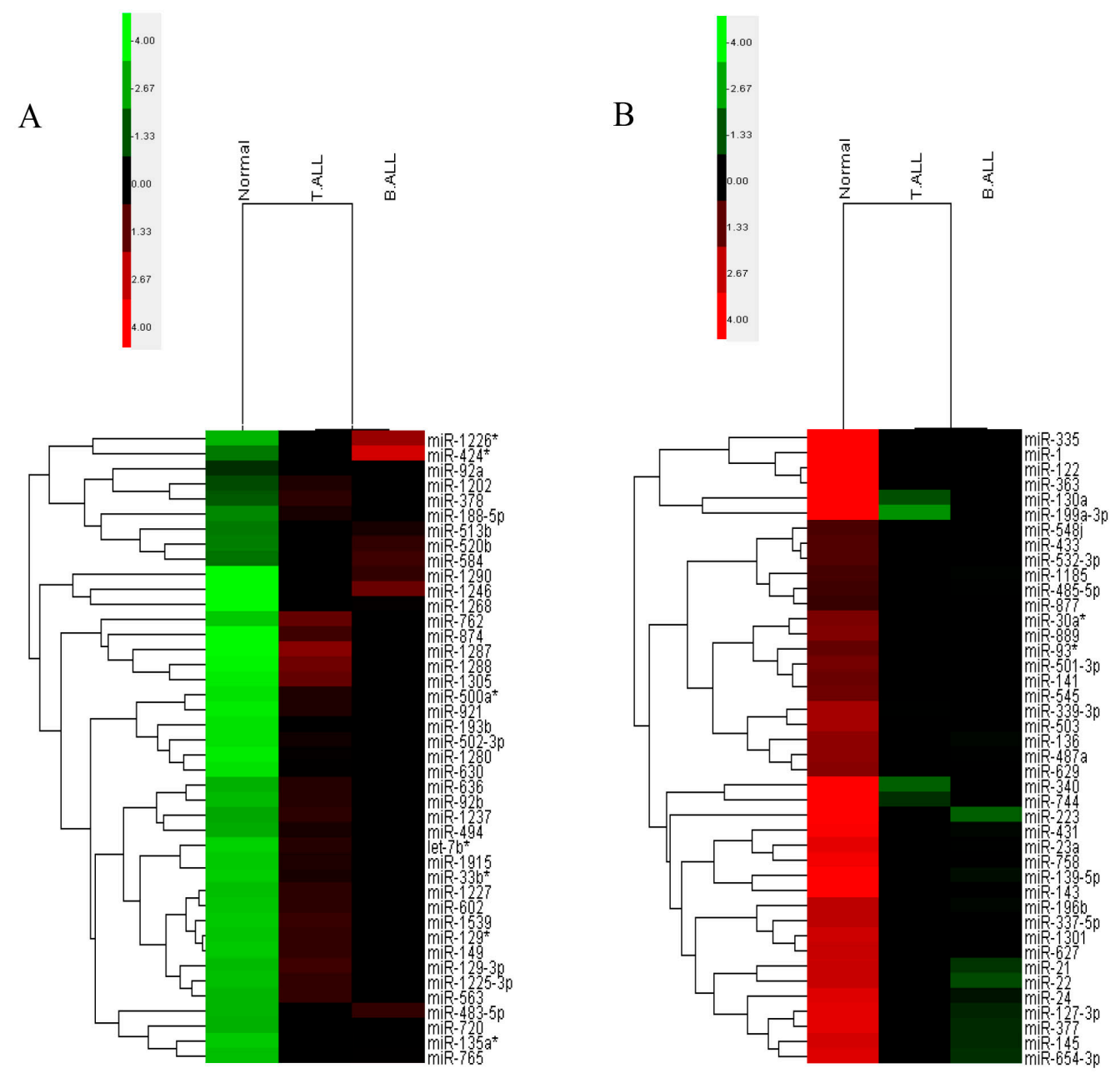

Figure 1. Heat-map of miRNA expression within circulating MVs from B-ALL, T-ALL and normal control subjects. A. Upregulated MV miRNAs in B-ALL and T-ALL groups relative to the normal control group. B. Downregulated MV miRNAs in B-ALL and T-ALL groups compared to the normal control group. MV= microvesicle; $\mathrm{B}-\mathrm{ALL}=\mathrm{B}$-cell acute lymphoblastic leukemia; T-ALL = T-cell acute lymphoblastic leukemia. 


\section{ALL-MVs enriched with abnormal miRNAs that regulate zinc finger protein (ZFP) genes}

From the analysis of the putative genes targeted by the aberrant MV miRNAs with TargetScan v6.2, we observed the interesting phenomenon that a large number of ZFP genes were regulated by these altered MV miRNAs. Specifically, 66 over-expressed miRNAs regulated 159 ZFP genes and 52 under-expressed miRNAs targeted 121 ZFP genes in B-ALL-derived MVs. In addition, of the aberrant miRNAs within T-ALL MVs, 59 upregulated miRNAs targeted 140 ZFP genes and 57 downregulated ones regulated 100 ZFP genes. Furthermore, among the miRNAs targeting ZFP genes, we found that 53 with high expression profiles and 50 with low expression signatures were shared between the two types of ALL-MVs (Table 4). ZFP genes regulated by the co-expressed MV miRNAs are listed in Table 5.

Table 4. Co-dysregulated miRNAs within the two types of ALL-derived MVs.

\begin{tabular}{ll}
\hline Co-upregulated miRNAs & Co-downregulated miRNAs \\
\hline miR-20b, miR-92a, miR-92b, miR-129-3p & miR-93, miR-23a, miR-23b, miR-361-5p \\
miR-130b, miR-133a, miR-149, miR-188-5p & miR-26a, miR-26b, miR-27a, miR-27b \\
miR-193b, miR-210, miR-296-5p, miR-345 & miR-30a, miR-30e, miR-148b, let-7f, let-7d \\
miR-365, miR-483-3p, miR-494, miR-501-5p & let-7a, let-7b, miR-98, miR-142-3p, miR-24 \\
miR-502-3p, miR-513a-5p, miR-513b, miR-550a & miR-7, miR-151-3p, miR-185, miR-454 \\
miR-557, miR-574-5p, miR-575, miR-601 & miR-301a, miR-16, miR-15b, miR-424, miR-374a \\
miR-622, miR-630, miR-634, miR-636 & miR-374b, miR-331-3p, miR-103, miR-107 \\
miR-654-5p, miR-665, miR-765, miR-767-3p & miR-32, miR-223, miR-221, miR-101, miR-486-5p \\
miR-874, miR-939, miR-940, miR-1202 & miR-21, miR-146a, miR-342-3p, miR-376a \\
miR-1227, miR-1228, miR-1229, miR-1234 & miR-150, miR-484, miR-425, miR-106b \\
miR-1237, miR-1238, miR-1246, miR-1260 & miR-22, miR-197, miR-362-5p, miR-18b \\
miR-1267, miR-1268, miR-1274a, miR-1274b & miR-15a, miR-186 \\
miR-1290, miR-1305, miR-1308 & \\
miR-1825, miR-1826 & \\
\hline
\end{tabular}

$\mathrm{ALL}=$ acute lymphoblastic leukemia; MVs = microvesicles.

Table 5. ZFP genes targeted by co-dysregulated miRNAs from the two ALL-MVs.

\begin{tabular}{ll}
\hline ZFP genes regulated by shared and upregulated miRNAs & ZFP genes targeted by common and downregulated miRNAs \\
\hline ZNF609, ZNF280B, ZNF714, ZNF385B, ZNF791 & ZNF274, ZNF275, ZNF689, ZNF831, ZNF566 \\
ZNF781, ZNF800, ZNF169, ZNF25, ZNF292 & ZNF644, ZNF217, ZNF711, ZNF410, ZNF622 \\
ZNF629, ZNF396, ZNF345, ZNF521, ZNF598 & ZNF597, ZNF572, ZNF322A, ZNF2, ZNF236 \\
ZNF451, ZNF740, ZNF81, ZNF713, ZNF295 & ZNF148, ZNF462, ZNF697, ZNF449, ZNF785 \\
ZNF770, ZNF280C, ZNF701, ZNF287, ZNF512B & ZNF267, ZNF81, ZNF609, ZNF295, ZNF280C \\
ZNF608, ZNF462, ZNF667, ZNF323, ZNF516 & ZNF667, ZNF238, ZNF25, ZNF420, ZNF280B \\
ZNF238, ZNF354A, ZFX, ZNF597, ZNF236 & ZNF772, ZNF512B, ZNF706, ZNF287, ZNF608 \\
ZNF2, ZNF3, ZNF24, ZNF131, ZNF148, ZNF329 & ZNF395, ZNF652, ZNF583, ZNF654, ZFP1 \\
ZNF200, ZNF207, ZNF217, ZNF655, ZNF697 & ZNF800 \\
ZNF644, ZNF512, ZNF518B, ZNF605, ZNF449 & ZBTB6, ZBTB11, ZBTB39, ZBTB10, ZBTB33 \\
ZFP28, ZFP62, ZFP91, ZFP106, ZNF36L1, ZNF36L2 & ZBTB44, ZBTB40, ZBTB4, ZBTB9, ZBTB34 \\
BCL11A, BCL11B, BCL6 & ZBTB5 \\
ZBTB34, ZBTB6, ZBTB9, ZBTB1, ZBTB43 & ZDHHC16, ZDHHC17, ZDHHC6, ZDHHC5 \\
ZBTB38, ZBTB20, ZBTB11, ZBTB41, ZBTB5 & ZDHHC7 \\
ZBTB2, ZBTB4, ZBTB26, ZBTB8, ZBTB7B & ZMYM2, ZMYM4 \\
ZC3H4, ZC3H7B, ZC3H7A, ZC3H12B, ZC3H14 & ZFYVE9, ZFYVE16, ZFYVE26 \\
ZC3H10, ZC3H11A & ZHX3, ZHX1 \\
ZFYVE9, ZFYV16, ZFYVE20, ZFYVE21 & ZXDA, ZXDB, ZXDC, TSHZ3, ZCCHC3, ZC3HAV1 \\
ZFYVE19, ZFYVE26 & ZFPM2, BAZ2B, GLIS2, VEZF1, GATAD2B, ST18 \\
ZDHHC16, ZDHHC18, ZDHHC21, ZDHHC22 & BCL11A, CTCF, ZDBF2, ZSCAN29, IKZF2, RCHY1 \\
TSHZ1, TSHZ3 & HELZ, RC3H2, CASZ1, ZFAND1, ZSWIM5 \\
ZFPM2, RBAK, BAZ2B, RCHY1, RC3H1, ST18 & IKZF2 \\
GLIS3, ZHX2, ZZZ3, GZF1, ZSCAN18, CNBP & \\
VEZF1, ZFHX4, ZRANB3, FIZ1, ZCRB1, ZSWIM1, ZC3HAV1L & \\
ZEB2, ZBED4, ZCCHC14, ZNFX1, ZMYM4, IKZF4 &
\end{tabular}

ALL-MVs = acute lymphoblastic leukemia microvesicles. 


\section{Target ZFP genes acted as oncogenes or tumor suppressive genes}

Considering that miRNAs play pivotal roles in regulating gene expression and have been implicated in cellular metabolism (Bartel, 2004), the potential target genes of dysregulated miRNAs might be related to disease pathogenesis. Concentrating on existing studies regarding ZFP genes, we observed that several ZFP genes were found to be involved in cell carcinogenesis (Table 6) or antineoplastic processes (Table 7). Among these, several, including ST18 and RCHY1, were targeted by both over- and under-expressed miRNAs. Furthermore, we found that ZFP genes that have been shown to be oncogenes were associated with upregulated MV miRNAs, and that ZFP genes shown to function as tumor suppressors were targeted by downregulated MV miRNAs.

Table 6. ZFP genes functioning as tumor-promoting factors regulated by altered MV miRNAs.

\begin{tabular}{|c|c|c|c|}
\hline MiRNAs & Gene & Description & Function \\
\hline $\operatorname{miR}-494$ & ZNF396 & zinc finger protein 396 & Repress Notch-Hes 1 signaling \\
\hline $\operatorname{miR}-1227$ & ZNF521 & zinc finger protein 521 & $\begin{array}{l}\text { Control of cell growth, clonogenicity, and } \\
\text { tumorigenic potential }\end{array}$ \\
\hline miR-940 & ZNF24 & zinc finger protein 24 & Promote cell growth, Wnt signaling pathway \\
\hline miR-130b, miR-24 & ZNF217 & zinc finger protein 217 & $\begin{array}{l}\text { Promote cell immortalization and invasion; attenuate } \\
\text { apoptotic signals }\end{array}$ \\
\hline $\begin{array}{l}\text { miR-557, miR-92a, } \\
\text { miR-92b, miR-103, miR-107 }\end{array}$ & BCL11A & $\begin{array}{l}\text { B-cell CLL/lymphoma } \\
11 \mathrm{~A} \text { (zinc finger protein) }\end{array}$ & $\begin{array}{l}\text { Candidate oncogene in lymphoid malignancies; } \\
\text { negatively regulate apoptosis }\end{array}$ \\
\hline $\operatorname{miR}-634$ & ZBTB20 & $\begin{array}{l}\text { zinc finger and BTB } \\
\text { domain containing } 20\end{array}$ & Promote TLR signaling, cell invasion, and metastasis \\
\hline $\operatorname{miR}-513 b$ & ZEB2 & $\begin{array}{l}\text { zinc finger E-box binding } \\
\text { homeobox } 2\end{array}$ & $\begin{array}{l}\text { E-cadherin repressor; induce cell } \\
\text { invasion and migration }\end{array}$ \\
\hline let-7a, let-7e, let-7f & ZNF689 & zinc finger protein 689 & Block pro-apoptotic signaling \\
\hline miR-26a, miR-26b & ZNF410 & zinc finger protein 410 & Proliferation, apoptosis, and cell cycle regulation \\
\hline miR-23a, miR-23b & ZNF267 & zinc finger protein 267 & Promote cell proliferation and migration \\
\hline miR-20b, miR-93 & ZBTB33 & $\begin{array}{l}\text { zinc finger and BTB } \\
\text { domain containing } 33\end{array}$ & $\begin{array}{l}\text { Repress E-cadherin expression; increase cell } \\
\text { migration and invasion }\end{array}$ \\
\hline miR-223 & ZNF706 & zinc finger protein 706 & $\begin{array}{l}\text { Cellular processes such as apoptosis, cell cycle, } \\
\text { DNA repair, and signal transduction }\end{array}$ \\
\hline miR-557, miR-494 & ZFX & $\begin{array}{l}\text { zinc finger protein, } \\
\text { X-linked }\end{array}$ & $\begin{array}{l}\text { Promote cell growth and migration; prevent } \\
\text { cell differentiation }\end{array}$ \\
\hline $\operatorname{miR}-630$ & ZNF451 & zinc finger protein 451 & Attenuate TGF- $\beta$-induced growth inhibitory responses \\
\hline miR-15b, miR-16, miR-103, miR-107 & HELZ & helicase with zinc finger & Bridge between SMYD3 and RNA polymerase II \\
\hline miR-149 & ZBTB2 & $\begin{array}{l}\text { zinc finger and BTB } \\
\text { domain containing } 2\end{array}$ & Inhibition of the $\mathrm{p} 53$ pathway \\
\hline miR-92a, miR-92b & ZFYVE21 & $\begin{array}{l}\text { zinc finger, FYVE } \\
\text { domain containing } 21\end{array}$ & $\begin{array}{l}\text { Regulate ECM degradation; promote cell metastasis } \\
\text { and invasion }\end{array}$ \\
\hline
\end{tabular}

$\mathrm{MV}=$ microvesicle; $\mathrm{CLL}=$ chronic lymphocytic leukemia; BTB = born to bind; FYVE = Fab1, YOTB, Vac1, $\mathrm{EEA} 1 ; \mathrm{ECM}=$ extracellular matrix.

\section{Bioinformatic analysis of ZFP genes regulated by MV miRNAs}

To explore the potential roles of the 207 ZFP genes targeted by the commonly altered MV miRNAs in cellular physiological and pathological processes, GO annotation was performed. GO terms were divided into three branches and each branch organized the genes into hierarchical categories with three layers. The GO terms with the highest enrichment and P values $<0.05$ are shown in Table 8 . It was obvious that the ZFP genes were predicted to primarily play roles in nucleic acid binding, transcription factor activity, intracellular part constitution, cell aging, and regulation of cellular metabolic process. We further investigated the ZFP geneassociated pathways using the Kegg, Biocarta, and National Cancer Institute (NCI)-Nature 
pathway databases, and found that the ZFP-associated signaling pathways $(\mathrm{P}<0.05)$ were correlated with DNA methylation, autophagy, protein catabolism, cell growth, apoptosis, and tumor progression (Table 9).

Table 7. ZFP genes functioning as antineoplastic factors targeted by abnormal MV miRNAs.

\begin{tabular}{|c|c|c|c|}
\hline miRNAs & Gene & Description & Function \\
\hline $\begin{array}{l}\operatorname{miR}-20 b, \operatorname{miR}-501-5 p \\
\text { miR-23a, miR-23b, } \\
\text { miR-26a, miR-26b, } \\
\text { miR-93, miR-361-5p }\end{array}$ & ZNF238 & zinc finger protein 238 & Decrease cell proliferation and promote cell death \\
\hline miR-133a & ZNF354A & zinc finger protein $354 \mathrm{~A}$ & Regulate cell proliferation \\
\hline miR-630, miR-20b, miR-93 & ZNF148 & zinc finger protein 148 & $\begin{array}{l}\text { Induce growth arrest and apoptosis; activation of } \\
\text { p21 (waf1) }\end{array}$ \\
\hline $\operatorname{miR}-767-3 p$ & ZFP64 & ZFP64 zinc finger protein & $\begin{array}{l}\text { Participate in Notch signaling; regulate cell } \\
\text { differentiation }\end{array}$ \\
\hline miR-1305, miR-188-5p & ZFP91 & ZFP91 zinc finger protein & $\begin{array}{l}\text { Regulator of NF- } \kappa B \text { signaling pathway, } \\
\text { cell proliferation, and anti-apoptosis }\end{array}$ \\
\hline miR-1246, miR-1825 & $\mathrm{ZC} 3 \mathrm{H} 10$ & $\begin{array}{l}\text { zinc finger } \mathrm{CCCH} \text {-type } \\
\text { containing } 10\end{array}$ & Inhibition of growth \\
\hline miR-502-3p, miR-223 & TSHZ3 & teashirt zinc finger homeobox 3 & $\begin{array}{l}\text { Novel tumor suppressor gene in breast and } \\
\text { prostate cancers }\end{array}$ \\
\hline miR-483-3p & RBAK & RB-associated KRAB zinc finger & $\begin{array}{l}\text { Cell cycle arrest; regulation of tumor suppressor } \\
\text { function of RB }\end{array}$ \\
\hline miR-130b, miR-148b & ST18 & $\begin{array}{l}\text { suppression of tumorigenicity } 18 \text {, } \\
\text { zinc finger }\end{array}$ & Apoptotic and inflammatory pathways \\
\hline $\operatorname{miR}-1260$ & ZHX2 & zinc finger and homeobox 2 & Repress cyclins A and E; inhibit cell proliferation \\
\hline miR-1305 & ZSCAN18 & $\begin{array}{l}\text { zinc finger and SCAN domain } \\
\text { containing } 18\end{array}$ & Candidate tumor suppressor gene \\
\hline miR-557, miR-345 & CNBP & $\begin{array}{l}\text { CCHC-type zinc finger, } \\
\text { nucleic acid binding protein }\end{array}$ & $\begin{array}{l}\text { Suppress cell metastasis and induce cell death; } \\
\text { regulation of c-myc transcription }\end{array}$ \\
\hline miR-15b, miR-16 & ZNF622 & zinc finger protein 622 & $\begin{array}{l}\text { Positive regulation of apoptosis signal-regulating } \\
\text { kinase } 1 \text { signaling }\end{array}$ \\
\hline $\operatorname{miR}-331-3 p$ & ZNF652 & zinc finger protein 652 & Directly repress key drivers of invasion and metastasis \\
\hline $\operatorname{miR}-27 a, \operatorname{miR}-27 b$ & ZHX1 & zinc fingers and homeoboxes 1 & Inhibit cell proliferation \\
\hline miR-26a, miR-26b & CASZ1 & castor zinc finger 1 & $\begin{array}{l}\text { Lengthen cell cycle progression; reprogram gene } \\
\text { expression }\end{array}$ \\
\hline
\end{tabular}

$\mathrm{MV}=$ microvesicle $\mathrm{RB}=$ retinoblastoma $\mathrm{KRAB}=$ Krüppel-associated box.

Table 8. Gene ontology (GO) annotation of target ZFP genes.

\begin{tabular}{llr}
\hline GO ID & Name & Hits \\
GO:0003676 & Molecular function & 138 \\
GO:0003702 & nucleic acid binding & 7 \\
GO:0016564 & RNA polymerase II transcription factor activity & 14 \\
GO:0003709 & transcription repressor activity \\
GO:0003682 & RNA polymerase III transcription factor activity & 9 \\
& ion binding & 164 \\
GO: 0043233 & Cellular component & 31 \\
GO:0043227 & organelle lumen & 139 \\
GO:0044424 & membrane-bounded organelle \\
GO:0030427 & intracellular part & 141 \\
GO: 43229 & site of polarized growth \\
& intracellular organelle & 15 \\
GO:0009058 & Biological process \\
GO:0044237 & biosynthetic process \\
GO:0007569 & cellular metabolic process \\
GO:0019222 & cell aging & 139 \\
GO:0007275 & regulation of metabolic process \\
\hline
\end{tabular}

\#Hits = the number of ZFP genes categorized into each GO term. 
Table 9. Analysis of target ZFP gene-associated signaling pathways.

\begin{tabular}{lll}
\hline miRNAs & Target genes & Pathway name \\
\hline miR-23a, miR-23b, miR-27a, miR-27b & CTCF & Mechanisms of transcriptional repression by DNA methylation \\
miR-20b, miR-130b, miR-550a & ZFYVE16, ZFYVE20, ZFYVE9 & Endocytosis \\
miR-1237 & BCL6 & Signaling events mediated by HDAC class II \\
miR-129-3p, miR-23a, miR-23b & RCHY1 & p53 signaling pathway \\
miR-20b, miR-130b & ZFYVE16, ZFYVE9 & TGF-beta signaling pathway \\
miR-129-3p, miR-23a, miR-23b & RCHY1 & Ubiquitin mediated proteolysis \\
miR-1290 & ZDHHC21 & eNOS activation and regulation \\
miR-1237 & BCL6 & IL-4-mediated signaling events \\
miR-1246, miR-96 & ZFP36L1 & C-MYC transcriptional repression \\
miR-1237 & BCL6 & FoxO family signaling \\
\hline
\end{tabular}

\section{DISCUSSION}

In this study, we investigated the expression profiles of miRNAs within MVs derived from two types of patients with ALL (B-ALL and T-ALL) and normal volunteers. Microarray results indicated that there were numerous aberrantly expressed MV miRNAs in the two kinds of ALL in comparison with normal controls. This, along with previous studies, indicated that miRNAs carried inside MVs were likely to be involved in ALL pathogenesis.

To explore the functions of altered MV miRNAs in ALL, we analyzed their putative target genes using TargetScan v6.2. We found that multiple ZFP genes were present in the lists of predicted target genes (Table 5), suggesting that ALL-MVs were enriched with distinct sets of miRNAs that regulated ZFP genes.

Proteins harboring zinc finger motifs were defined as ZFPs. Previous studies had clarified the basic unit of a zinc finger motif to contain a finger-like secondary structure acting as a DNA/RNA-binding domain. ZFPs were shown to be important transcription factors and play vital functions in regulating growth, development, activation, apoptosis, and other processes (Leon and Roth, 2000). Dysregulation of the miRNAs targeting ZFP might lead to their altered expression and loss of control of related cellular metabolic processes such as cell immortalization and apoptosis, eventually leading to the occurrence of cellular carcinogenesis. Furthermore, MVs are known as a class of novel carriers having crucial roles in intercellular communication (Valadi et al., 2007). Therefore, it might be expedient for miRNAs packaged inside ALL-MVs to play regulatory functions through being horizontally transferred to surrounding locations in the ALL microenvironment. Our results showed that ALL-MVs were equipped with both up- and downregulated miRNAs targeting ZFP genes. Therefore, in leukemia microenvironmental niches, ALL-MVs might transfer these aberrantly expressed miRNAs to their target cells to abnormally regulate the expression of ZFP genes that might possibly played roles in the progression of ALL.

It has been documented that some members of the zinc finger family have close relationships with pathophysiologic mechanisms in cancer. For example, expression of zinc finger protein 238 (ZNF238) drastically repressed brain tumor cell growth and promoted cell death and regulated cell cycle arrest (Tatard et al., 2010). Furthermore, functional analysis indicated that ZNF267 increased the proliferation rate and migration of HCC cells in vitro (Schnabl et al., 2011). In this study, ZNF238 was targeted by over-expressed miR-20b, which was reported to be an oncogenic miRNA (Li et al., 2013). Conversely, ZNF267 was a target gene of the antineoplastic miRNAs -23a and -23b (Goto et al., 2014; Xishan et al., 2014), both of which were downregulated in our study. Given that the role of miRNAs generally lies in 
negatively regulating target gene expression, $\mathrm{miR}-20 \mathrm{~b}$ might be positively correlated with leukemogenesis by directly inhibiting the expression of the tumor suppressive ZNF238 protein. Likewise, ZNF267 was likely to participate in the tumorigenic activities of ALL by the fact that it was modulated by the downregulated miRNAs $-23 \mathrm{a}$ and $-23 \mathrm{~b}$. In addition, we identified that several oncogenic and antineoplastic ZFP genes were targeted by high- and low-expressed MV miRNAs, respectively, indicating that MV miRNAs and their target ZFP genes in different microenvironments might participate in disparate cellular metabolic processes, eventually producing specific effects on cells.

We further investigated the biological roles of the target ZFP genes using informatic tools. GO annotation demonstrated that most targeted ZFP genes are predicted to be involved in the regulation of cellular metabolism, such as growth and aging. Therefore, these target genes might directly exert influences on the survival capability of leukemia cells. Using pathway database analysis, ring finger and $\mathrm{CHY}$ zinc finger domain containing 1 (RCHY1) and zinc finger, FYVE domain containing 16 (ZFYVE16) were shown to be independently involved in the p53 and TGF-beta signaling pathways, respectively. It is well-known that the two pathways are associated with the regulation of cell proliferation, differentiation, apoptosis, and the cell cycle, and they have been reported to contribute cancer development (Prives, 1998; Massagué, 2008). In this study, RCHY1 was targeted by the downregulated miRNAs -23a and -23b, and ZFYVE16 was modulated by the upregulated miR-20b. Thus, p53 and TGF-beta pathways might have their normal functions compromised owing to the altered expression of ZFP genes in their respective pathway systems, eventually leading to the abnormal biological characteristics of ALL cells such as failure of differentiation and disordered apoptosis. These findings suggested that cancerassociated MV miRNAs and their target ZFP genes might be novel pathogenic factors in ALL. These conclusions, however, require further validation.

In conclusion, we demonstrated for the first time that ALL-MVs were enriched with dysregulated miRNAs, a large proportion of which targeted ZFP genes, indicating that miRNAs regulating ZFP genes were active in ALL-MVs. Furthermore, our results suggested that ZFP genes regulated by distinct sets of miRNAs with that had undergone specific expression changes were likely to participate in the molecular pathological mechanisms that contribute to the progression of ALL.

\section{ACKNOWLEDGMENTS}

Research supported by the National Nature Science Foundation of China (Grant \#81170462).

\section{REFERENCES}

Bartel DP (2004). MicroRNAs: genomics, biogenesis mechanism, and function. Cell 116: 281-297.

Cocucci E, Racchetti G and Meldolesi J (2009). Shedding microvesicles: artefacts no more. Trends Cell Biol. 19: 43-51.

Ferlay J, Soerjomataram I, Ervik M, Dikshit R, et al. (2013). GLOBOCAN 2012 v1.0, cancer incidence and mortality worldwide: IARC Cancer Base No. 11 [Internet]. International Agency for Research on Cancer, Lyon. Available at [http://globocan.iarc.fr] Accessed September 30, 2014.

Goto Y, Kojima S, Nishikawa R, Enokida H, et al. (2014). The microRNA-23b/27b/24-1 cluster is a disease progression marker and tumor suppressor in prostate cancer. Oncotarget 5: 7748-7759.

Guo H, Ingolia NT, Weissman JS and Bartel DP (2010). Mammalian microRNAs predominantly act to decrease target mRNA levels. Nature 466: 835-840.

Ikeda S and Tagawa H (2014). Dysregulation of microRNAs and their association in the pathogenesis of T-cell lymphoma/ 
leukemias. Int. J. Hematol. 99: 542-552.

Leon O and Roth M (2000). Zinc finger: DNA binding and protein-protein interaction. Biol. Res. 33: 21-30.

Li D, Ilnytskyy Y, Kovalchuk A, Khachiqian LM, et al. (2013). Crucial role for early growth response-1 in the transcriptional regulation of miR-20b in breast cancer. Oncotarget 4: 1373-1387.

Li WY, Chen XM, Xiong W, Guo DM, et al. (2014). Detection of microvesicle miRNA expression in ALL subtypes and analysis of their functional roles. J. Huazhong Univ. Sci. Technolog. Med. Sci. 34: 640-645.

Massagué J (2008). TGFbeta in Cancer. Cell 134: 215-230.

Negrini M, Nicoloso MS and Calin GA (2009). MicroRNAs and cancer - new paradigms in molecular oncology. Curr. Opin. Cell Biol. 21: 470-479.

Pisetsky DS, Gauley J and Ullal AJ (2011). Microparticles as a source of extracellular DNA. Immunol. Res. 49: 227-234. Prives C (1998). Signaling to p53: breaking the MDM2-p53 circuit. Cell 95: 5-8.

Pui CH, Robison LL and Look AT (2008). Acute lymphoblastic leukemia. Lancet 371: 1030-1043.

Raposo G and Stoorvogel W (2013). Extracellular vesicles: exosomes, microvesicles, and friends. J. Cell. Biol. 200: 373-383.

Schnabl B, Valletta D, Kirovski G and Hellerbrand C (2011). Zinc finger protein 267 is up-regulated in hepatocellular carcinoma and promotes tumor cell proliferation and migration. Exp. Mol. Pathol. 91: 695-701.

Srivastava SK, Arora S, Singh S, Bhardwaj A, et al. (2014). MicroRNAs in pancreatic malignancy: progress and promises. Cancer Lett. 347: 167-174.

Tatard VM, Xiang C, Biegel JA and Dahmane N (2010). ZNF238 is expressed in postmitotic brain cells and inhibits brain tumor growth. Cancer Res. 70: 1236-1246.

Valadi H, Ekström K, Bossios A, Sjöstrand M, et al. (2007). Exosome-mediated transfer of mRNAs and microRNAs is a novel mechanism of genetic exchange between cells. Nat. Cell Biol. 9: 654-659.

Vitale A, Guarini A, Chiaretti S and Foà R (2006). The changing scene of adult acute lymphoblastic leukemia. Curr. Opin. Oncol. 18: 652-659.

Xishan Z, Xianjun L, Ziying L, Guangxin C, et al. (2014). The malignancy suppression role of miR-23a by targeting the BCR/ABL oncogene in chronic myeloid leukemia. Cancer Gene Ther. 21: 397-404.

Zhang HG and Grizzle WE (2014). Exosomes: a novel pathway of local and distant intercellular communication that facilitates the growth and metastasis of neoplastic lesions. Am. J. Pathol. 184: 28-41. 